\title{
Radiological Findings In Thalassemia
}

The Mediterranean autosomal recessive microcystic is anemia aka thalassemia, is a disorder of haemoglobin synthesis with decreased production of either alpha or beta polypeptide chains of haemoglobin molecules which are termed as alpha-thalassemia or beta-thalassemia respectively. It exists in two forms - homozygous (thalassemia major) and heterozygous (thalassemia minor and thalassemia minima). Thalassemia major causes severe anemia in infants and young children with a high mortality. Bony changes leading to rodent-like facies along with hepatosplenomegaly can be seen. The thalassemia trait may confer a degree of protection against malaria, which gives a selective survival advantage to carriers.

Plain radiographs usually suffice to confirm the diagnosis. The complete Skeletal system is involved. The marrow proliferation causes the medulla to enlarge relative to the cortex, leading to bony expansion and cortical thinning along with resorption of cancellous bone resulting in generalized loss of bone density. In long bones, the characteristic Erlenmeyer flask appearance is noted though not specific to the condition. Within the medulla, first there is thinning of the trabeculae followed by their coarsening, the changes being most marked in the metacarpals and phalanges, which become cylindrical or biconvex in shape. Fractures may occur, although less commonly seen than expected from the degree of osteoporosis. Well-defined erosions of the periosteal margin of the cortex of the $n$ metaphysis or diaphysis may be identified in severe cases. In severe cases, the paranasal sinuses develop poorly and may contain red marrow leading to facial abnormalities and dental malocclusion. The ethmoidal cells are spared since they lack red marrow.

The classic "hair-on-end" appearance, seen on lateral skull $x$-rays, most severely noted in the frontal bone are the earliest changes in the skull vault due to widening of diploic space, thinning of the tables, frequently with complete obliteration of the outer table and new bone formation in the form of bony spicules in response to marrow proliferation beneath the periosteum. A simple chest $\mathrm{x}$-ray may show cardiomegaly and signs of congestive heart failure which mirrors the degree of anaemia and also cortical erosions as well as club-like anterior ends in the ribs. A frequent finding is the "rib-within-a-rib appearance" due to subperiosteal extension of haemopoietic tissue through the rib cortex and is noted particularly in its middle and anterior portions. Spinal changes noted in anteroposterior and lateral view of thoracolumbar spine may consist of generalised osteopenia, resulting in compression fractures and biconcavity of the vertebral bodies. Scoliosis is a common finding in thalassemia major patients with platyspondyly reported as a complication of hypertransfusion. Short stature may result due to premature fusion of growth plates. Desferoxamine therapy can also lead to morphological changes in the long bone metaphyses. In the less severe variants, diffuse osteopenia may be the only radiological finding.

Extramedullary hematopoiesis occurs in severe cases surviving to adulthood. The common sites are thoracic paravertebral region by extension from the adjacent ribs, the mediastinum and presacral space. Conventional radiography may fail to show such changes in early state, which is best demonstrated on magnetic resonance imaging (MRI) showing diffuse reduction in marrow signal intensity on T1-weighted images due to marrow reconversion. Quantitative MRI is an accurate noninvasive method for assessing hepatic iron concentration, reducing the need for liver biopsy.

Computed tomography (CT) scanning may aid in further evaluation of the faciomaxillary changes and to delineate the expansile lesions of the pelvis due to extramedullary hematopoiesis. CT scanning confirms the deposition of iron in liver and other organs but this finding is not consistent with organ damage, serum ferritin levels and transfusional history. Ultrasonography is the modality of choice for cholelithiasis, which is a common finding seen in untreated thalassemia. It can also easily-depict changes such as hepatosplenomegaly. Most of the skeletal changes can be prevented with early blood transfusion therapy but iron overload remains a possibility. 